\title{
Pulmonary vascular disease in Fontan circulation - is there a rationale for pulmonary vasodilator therapies?
}

\author{
Kolja Becker ${ }^{1}$, Anselm Uebing ${ }^{1,2}$, Jan Hinnerk Hansen ${ }^{1,2}$ \\ ${ }^{1}$ Department of Congenital Heart Disease and Pediatric Cardiology, University Hospital of Schleswig-Holstein, Campus Kiel, Kiel, Germany; \\ ${ }^{2}$ DZHK (German Center for Cardiovascular Research), Partner Site Hamburg/Kiel/Lübeck, Germany \\ Contributions: (I) Conception and design: All authors; (II) Administrative support: All authors; (III) Provision of study materials or patients: All \\ authors; (IV) Collection and assembly of data: All authors; (V) Data analysis and interpretation: All authors; (VI) Manuscript writing: All authors; (VII) \\ Final approval of manuscript: All authors. \\ Correspondence to: Kolja Becker. Department of Congenital Heart Disease and Pediatric Cardiology, University Hospital of Schleswig-Holstein, \\ Campus Kiel, Arnold-Heller-Strasse 3, 24105 Kiel, Germany. Email: kolja.becker@uksh.de.
}

\begin{abstract}
The Fontan circulation is a palliative concept for patients with univentricular hearts. The central veins are connected directly to the pulmonary arteries (cavo-pulmonary connection) to separate the pulmonary and the systemic circulation. There is no sub-pulmonary ventricle that generates pressure to drive blood through the pulmonary arteries. Pulmonary blood flow is determined by central venous pressure (CVP) and pulmonary vascular resistance (PVR). The capability of the Fontan circulation to compensate for alterations in PVR is limited, as CVP can only be increased within narrow ranges without adverse clinical consequences. Consequently, systemic ventricular preload and cardiac output are dependent on a healthy lung with low PVR. Failure of the Fontan circulation is relatively common. In addition to ventricular dysfunction, maladaptive pulmonary vascular remodeling resulting in increased pulmonary resistance may play a key role. The pathophysiology of the maladaptive vascular processes remains largely unclear and diagnosis of an increased PVR is challenging in Fontan circulation as accurate measurement of pulmonary arterial blood flow is difficult. In the absence of a sub-pulmonary ventricle, pulmonary artery pressure will almost never reach the threshold conventionally used to define pulmonary arterial hypertension. There is a need for markers of pulmonary vascular disease complementary to invasive hemodynamic data in Fontan patients. In order to treat or prevent failure of the Fontan circulation, pathophysiological considerations support the use of pulmonary vasodilators to augment pulmonary blood flow and systemic ventricular preload and lower CVP. However, to date the available trial data have neither yielded enough evidence to support routine use of pulmonary vasodilators in every Fontan patient nor have they been helpful in defining subgroups of patients that might benefit from such therapies. This review discusses potential pathomechanisms of pulmonary vascular disease; it summarizes the current knowledge of the effects and efficacy of pulmonary vasodilator therapy in Fontan patients and tries to outline areas of potential future research on the diagnosis and treatment of pulmonary vascular disease and Fontan failure.
\end{abstract}

Keywords: Fontan circulation; hypoxia-inducible factor 1; pulmonary hypertension; univentricular heart; vasodilators

Submitted Apr 12, 2020. Accepted for publication Jun 29, 2020.

doi: $10.21037 / \mathrm{cdt}-20-431$

View this article at: http://dx.doi.org/10.21037/cdt-20-431 


\section{The Fontan procedure}

The basic principle of the Fontan circulation is that the systemic venous return passes through the pulmonary circulation without the driving force of ventricular contraction, while the single functional ventricle supports the systemic circulation. In the current era, the creation of a total cavo-pulmonary connection (TCPC) is the surgical technique of choice. The Fontan procedure places the pulmonary and systemic circulation in series and, in this way, reduces cyanosis (Figure 1).

The Fontan procedure with its variants has been used as a palliative strategy for patients with a single functional ventricle for more than 45 years. Outcomes of single ventricle patients undergoing a Fontan operation improved over the past decades. Patient selection and introduction of staged surgical management as well as refinements in surgical and postoperative care resulted in low perioperative mortality. Nowadays, most patients undergoing a Fontan procedure will survive into adulthood (1), but mortality is estimated to increase by about $10 \%$ per decade (2). It can be expected that less than two thirds of the early survivors of the Fontan procedure will reach their forties or fifties (2).

\section{The Fontan physiology}

In the Fontan circulation, pulmonary blood flow is mainly driven by central venous pressure (CVP) as there is no subpulmonary ventricle. Based on physiological considerations, there is a linear relationship between CVP and pulmonary vascular resistance (PVR), which also has been supported with clinical data (3). As a consequence, a mild increase in PVR needs to result in a marked increase in CVP to maintain cardiac output (3) (Figure 2). Consequently, low PVR is necessary to sustain adequate pulmonary blood flow at low CVP. However, CVP is invariably elevated even in well-functioning Fontan patients with an inherent risk of complications related to chronic venous hypertension and circulatory failure $(4,5)$.

The ability to increase pulmonary blood flow and in turn

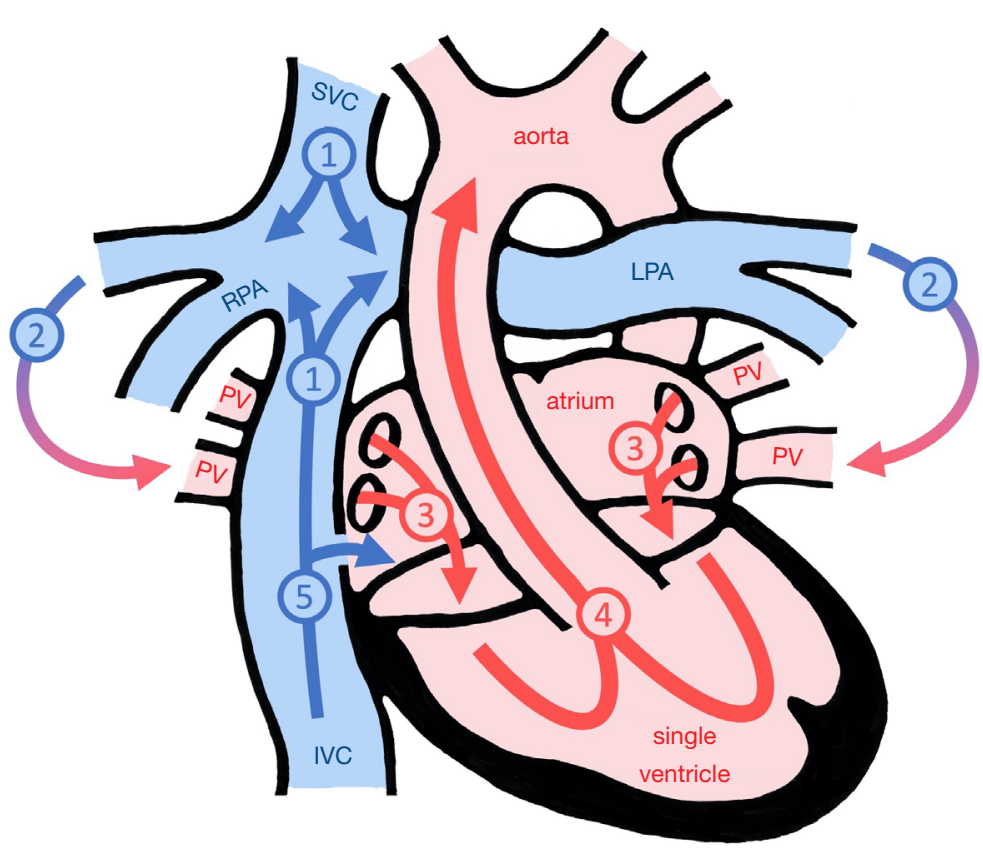

Figure 1 The Fontan circulation. The Fontan procedure serves as a palliative procedure for a variety of complex congenital heart defects with a functional single ventricle. Systemic venous blood from the superior vena cava (SVC) and the inferior vena cava (IVC) drains directly into the left and right pulmonary arteries (LPA and RPA) (1). Pulmonary blood flow is passive and is mainly driven by central venous pressure (CVP) (2). Pulmonary veins (PV) drain the oxygenated blood into the atrium (3). Pulmonary venous return provides filling of the single ventricle. Ventricular preload is dependent upon flow through the pulmonary circulation (4). A patent fenestration in the Fontan tunnel results in a right-to-left shunt on the atrial level, which might improve preload of the ventricle but on the expense of enhanced cyanosis (5). 


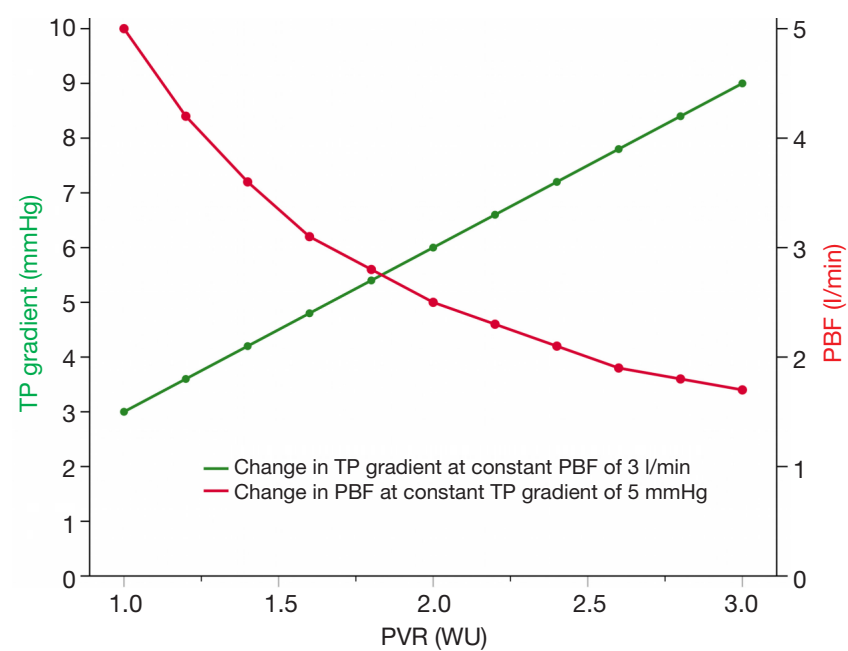

Figure 2 Relation between pulmonary blood flow (PBF), pulmonary vascular resistance (PVR) and central venous pressure (CVP). In theory, there is a strong linear relationship between CVP and transpulmonary gradient (TP). If PVR increases in Fontan patients, the TP will increase if pulmonary blood flow is constant (green line). Changes of CVP in relation to PVR are dependent on $\mathrm{PBF}$. Small increments in PVR can result in a significant reduction of PBF (red line). In extreme cases, CVP can be in a normal range for Fontan patients with low cardiac output despite elevated PVRi.

oxygen consumption during exercise is limited in Fontan patients (6). Exercise tolerance is reduced already early after Fontan completion (7) and progressively declines with age $(8,9)$. The inability to decrease PVR during exercise is a contributor to this $(10,11)$. This phenomenon can at least partly be explained by the absence of pulsatile pulmonary arterial flow, which is believed to acutely recruit pulmonary capillaries during exercise in subjects with a biventricular circulation (12).

If there is a surgically created communication between the central venous compartment and the functionally left atrium (Fontan fenestration), systemic venous blood can bypass the lungs and improve preload of the systemic ventricle. But this is on the expense of enhanced cyanosis (Figure 1). While there is some evidence that a Fontan fenestration improves early postoperative outcome $(13,14)$, there is little evidence that a patent fenestration improves functional status in the longer-term $(15,16)$. It may well be that the effect of blood flow through a fenestration on ventricular filling and systemic cardiac output is counterbalanced by increasing cyanosis from right-to-left shunting resulting in no net effect on tissue oxygenation.

Before a cavo-pulmonary connection is established in patients with univentricular hearts, the single ventricle is commonly exposed to abnormal loading conditions (17). The pulmonary and systemic circulations are supplied in parallel by the single ventricle. In malformations where pulmonary blood flow is increased ventricular volume overload can be excessive. Surgical aortic arch reconstruction (e.g., in patients with hypoplastic left heart syndrome undergoing the Norwood operation) results in impaired aortic elasticity and increased ventricular afterload. These abnormal loading conditions, which are often longstanding and present in combination, may lead not only to systolic, but also to diastolic ventricular dysfunction (18). The latter adds to the inherent inefficacy of the Fontan circulation and worsens central venous congestion (3).

It becomes apparent from the above that the Fontan circulation is prone to fail. Systolic and/or diastolic ventricular dysfunction, obstructions in the Fontan pathways, elevated PVR all aggravate chronic systemic venous hypertension and lead to symptoms of heart failure such as ascites, peripheral edema and hepatomegaly or result in specific complications related to lymphatic dysfunction such as plastic bronchitis or protein-losing enteropathy $(19,20)$. Signs of maladaptation to the Fontan circulation can develop in nearly every organ system (21), with hepatic and renal disease being particularly common in adult Fontan patients (19). Treatment options for failing Fontan patients are limited and include conventional heart failure therapies, Fontan conversion to improve venous fluid dynamics, interventions that aim to decompress the systemic venous compartments such as creation of Fontan fenestrations and more recently pulmonary vasodilator therapies aiming at reduction of PVR.

\section{The pulmonary vasculature in Fontan patients}

It is not possible to establish a Fontan circulation in a neonate as PVR is still elevated after birth. Therefore, many patients will experience a period of increased pulmonary blood flow (and sometimes increased pressure) either through a systemic-to-pulmonary shunt or a variably obstructed pulmonary artery before a cavo-pulmonary connection can be established. During this time period, increased pulmonary blood flow may enhance pulmonary artery growth (17). On the other hand, pulmonary overcirculation may lead to irreversible changes in pulmonary vascular morphology with an increase in PVR (22). Thus, the pulmonary artery tree of single ventricle patients may already be diseased prior to the Fontan procedure-either 
as a result of reduced pulmonary blood flow resulting in a diminished pulmonary vascular cross-sectional area, or as a result of increased pulmonary flow and pressure resulting in adverse vascular remodeling. Both scenarios may leave the lung with an abnormal PVR and are a burden for the Fontan circulation that is to be established.

Accurate determination of PVR is difficult in the Fontan circulation. Hemodynamic studies performed under general anesthesia with a positive end-expiratory pressure might result in misleading central venous and pulmonary arterial pressure measurements. Collateral blood flow, residual antegrade flow across a patent pulmonary valve or a patent fenestration complicates quantification of pulmonary blood flow and in turn estimation of PVR. The most precise estimation of PVR might be achieved by combining cardiac MRI pulmonary blood flow measurement and invasive pressure measurements $(23,24)$. However, combined cardiac catheterization and MRI suites are not universally available.

Pulmonary artery pressure in the Fontan circulation will almost never reach the threshold used to define pulmonary hypertension in patients with a biventricular circulation where mean pulmonary artery pressures $>20 \mathrm{mmHg}$ are defined as elevated according to the most recent definition from the 6th World Symposium on Pulmonary Hypertension held in Nice in 2018 (25). In Fontan patients a transpulmonary gradient $>6 \mathrm{mmHg}$ and a PVR index $>3 \mathrm{WU} \times \mathrm{m}^{2}$ has been suggested as a definition for pulmonary hypertensive vascular disease by the European Pediatric Pulmonary Vascular Disease Network (EPPVDN) (26). If the EPPVDN-definition is applied, data published by Khambadkone and co-workers showed that one decade after their Fontan operation only a very small proportion of Fontan patients with good functional status have an elevated PVR based on invasive hemodynamic data (27). In contrast, Mitchell and colleagues found that PVR was elevated in most patients with overt Fontan failure after heart transplantation (28). Autopsy studies from patients with a long-standing Fontan circulation demonstrated pulmonary vascular remodeling characterized by intimal thickening and changes in vascular smooth muscle cells $(22,29)$. It has been speculated that the Fontan circulation itself initiates and maintains these maladaptive processes within in the lung. However, the underlying processes and mechanisms are still poorly understood.

\section{Abnormal pulmonary blood flow in Fontan patients}

The most pervasive hypothesis is that the lack of pulsatile blood flow plays a major role in the development of pulmonary vascular disease in Fontan patients. Pulsatile pulmonary arterial flow is responsible for shear stress mediated release of endothelium derived vasoactive factors in a biventricular circulation. Several studies have shown that the absence of pulsatile pulmonary artery flow is associated with pulmonary vascular changes in animals with a single-lung Glenn-anastomosis and in patients with a bidirectional Glenn-anastomosis and that these changes are mitigated by residual pulsatile flow (30-33). Zongtao and co-workers showed particularly that the expression of endothelial nitric oxide synthetase (eNOS) was significantly reduced with nonpulsatile flow (30). The eNOS is a vasoactive factor synthesized and secreted by pulmonary endothelial cells, it controls the bioavailability of nitric oxide (NO). A state in which eNOS enzymatic activity is attenuated or altered and NO bioavailability decreased is referred to as endothelial dysfunction (34). It is characterized by alterations in vascular tone and pulmonary vascular remodeling. Accordingly, Latus et al. demonstrated that pulmonary flow reserve was reduced in Fontan patients (35), as these showed an abnormal response to acetylcholine, whose action on vascular tone is mediated mainly by NO.

If absence of pulsatile pulmonary blood flow and the resulting endothelial dysfunction would be the only trigger of elevated PVR in Fontan patients, the underlying pathophysiology would be confined to the pulmonary vascular endothelium. However, absence of pulsatile blood flow does not only impact on the affected lung. Sage et al. compared piglets with either a ligated right pulmonary artery or a right pneumonectomy (36). They were able to show that pulmonary artery ligation induced changes in vascular function and morphology in the contralateral lung. These changes were absent in the pneumonectomy group. These results suggest that the lung with absent pulmonary blood flow released factors that were responsible for vascular remodeling in the contralateral lung.

In addition, absence of pulsatile blood flow is not the only factor, which leads to pulmonary vascular changes in single ventricle patients. Patients with cavo-pulmonary connections that lack hepatic venous blood flow to the lung (e.g., after the Kawashima-procedure) and animal models with a classical Glenn-Anastomosis are prone to develop pulmonary arterio-venous malformations, which usually regress when hepatic venous blood flow to the lung is restored $(32,37)$. It has been hypothesized from this observation that the absence of a growth-modulating endocrine factor produced in the liver may alter the activity 
of vascular endothelial growth factor (VEGF) or endothelin in Glenn- or Kawashima-patients $(38,39)$.

\section{Hypoxia inducible factors (HIF) in Fontan patients}

Hypoxia is defined as inadequate tissue oxygen tension caused by interplay of hypoxemia, low cardiac output, insufficient tissue vascularization, anemia and other factors. Each cell responds to the hypoxic environment with enhanced expression of a group of genes that are hypoxia inducible. Their hypoxic induction is mediated by the HIF (HIF-1 $\alpha$ and HIF-2 $\alpha$, referred to simply as HIF in the following paragraphs) (40). Among others, HIF is targeting genes coding for erythropoietin, endothelin-1 and VEGF (41). Endothelin is a potent vasoconstrictor and is involved in vascular inflammation and pulmonary arterial hypertension, which is why endothelin-receptor antagonists are widely used to treat pulmonary arterial hypertension. VEGF is an angiogenetic factor whose role in pulmonary arterial hypertension is not completely understood, but there is growing evidence that VEGF plays an important role in the pathogenesis of pulmonary arterial hypertension (42).

According to the afore mentioned definition, most Fontan patients must be regarded as chronically hypoxic. While the role of HIF and target genes is well characterized in hematology and oncology (43), its role in Fontan patients is still unclear. Erythropoiesis seems to be increased despite of insufficient iron stores in Fontan patients (44), which is most likely a result of elevated erythropoietin levels. Mori et al. showed that circulating angiogenetic growth factor levels (e.g., VEGF) are increased in Fontan patients (45). Suda and colleagues found that VEGF levels are elevated in cyanotic patients and stay elevated in patients with a univentricular heart even after Fontan completion (46). There is immunohistochemical evidence of increased levels of endothelin-1 (47) and the endothelin-receptor in the pulmonary arteries of failed Fontan patients (48) and there have been reports of elevated circulating plasma levels of endothelin-1 in early post-operative Fontan patients $(49,50)$. Therefore, as the levels of HIF-regulated denominators of PVR (i.e., VEGF and endothelin-1) seem to be increased in Fontan patients, there is some evidence suggesting that the Fontan circulation is a state of chronic HIF activation. In addition to endothelial dysfunction, chronic HIF activation may be another maladaptive process adversely impacting on PVR in Fontan patients. HIF activation may even be a modulator of endothelial dysfunction, as NO-production is critically regulated by arginase-1, an enzyme whose induction is HIF-mediated.

While direct evidence is still scarce that levels of HIFregulated gene products are chronically increased in Fontan patients and that HIF activation is contributing to their pulmonary vascular changes, observations in organ systems other than the lung also suggest that HIF activation is one of the characteristic features of the Fontan circulation (51). It is remarkable that for example pheochromocytoma and retroperitoneal paraganglioma have been reported to occur with an unusually high incidence in Fontan patients $(52,53)$. In non-cyanotic cases these rare neuroendocrine tumors are often associated with chronic activation of the HIF-pathway caused by genetic mutations-one might speculate that in Fontan patients chronic HIF activation caused by tissue hypoxia promotes development of these tumors. Another example is that hepatocellular carcinoma is found in Fontan patients with Fontan associated liver disease at a rather early age $(54,55)$ which is not unlikely also due to chronic tissue hypoxia in the Fontan circulation, as it is well known that hypoxia and HIF play a significant role in the development of hepatocellular carcinoma (56).

HIF activation is counterbalanced by several pathways. One of them-the prolyl-hydroxylase-pathway-is dependent on iron as a cofactor (57). There is a complex interaction between iron status, HIF expression and HIF degradation. Iron supplementation can partially reverse pulmonary hypertension while iron depletion can aggravate pulmonary hypertension caused by hypoxia (58-60). In can be speculated from the above that the adverse effects of iron depletion on heart failure symptoms in Fontan patients are not only related to anemia and the resulting reduction in oxygen carrying capacity but also to its adverse effects on the pulmonary vasculature via reduced HIF inactivation.

To date, it remains speculative which molecules are promising targets for HIF-modulation in Fontan patients and if any of these potential therapies will improve outcome. Further research into the potential role of HIF activation and VEGF activation in the development of pulmonary vascular disease in Fontan patients is necessary to develop new target specific therapies that modulate PVR in this complex and vulnerable patient group (61-63) (Figure 3).

\section{Pulmonary vascular disease in Fontan failure}

Fontan failure is a term used for a devastating scenario that sets apart Fontan patients from all other patients with congenital heart disease. It is characterized by protein losing enteropathy, plastic bronchitis, chronic (often chylous) 


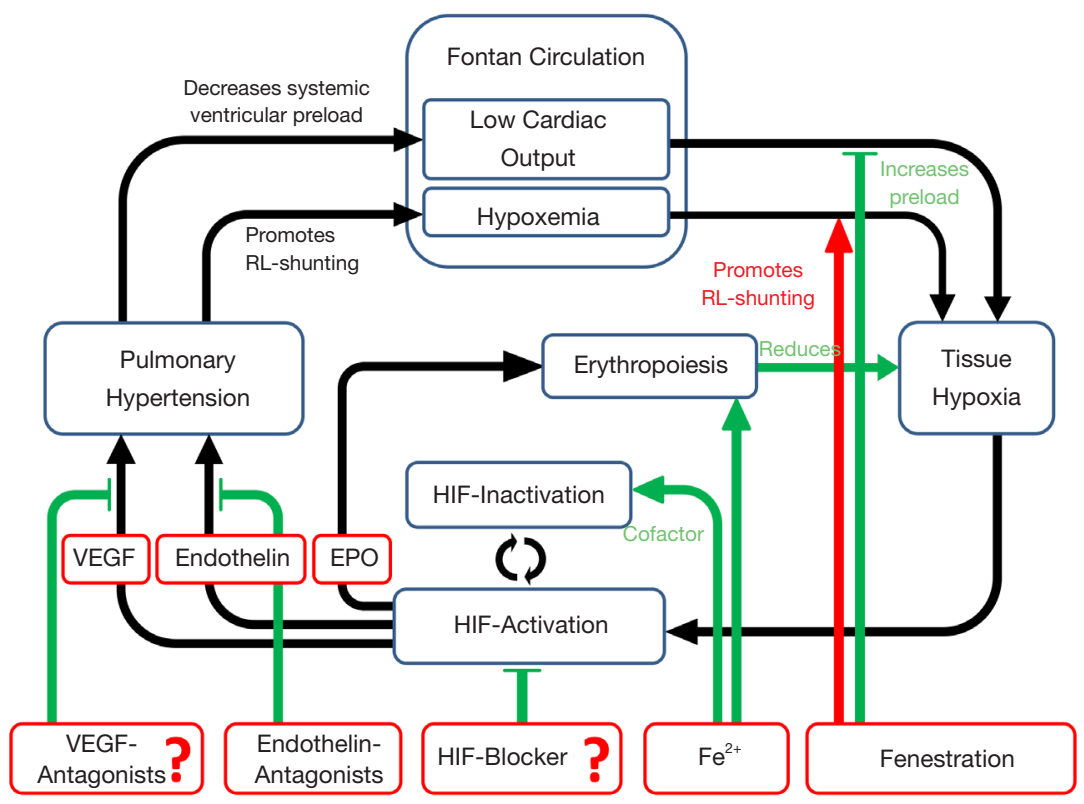

Figure 3 Model of the mechanisms that likely initiate and maintain pulmonary vascular changes in Fontan patients. Green lines and arrows depict effects that might ameliorate and red lines and arrows effects that likely worsen pulmonary vascular disease in Fontan patients. Possible therapeutic strategies that focus on these mechanisms are highlighted with red boxes on the bottom of the figure.

pleural effusions, ascites or Fontan associated liver or renal disease. The term can also be used more broadly for Fontan patients with a declining clinical status, reduced healthrelated quality of life and end stage heart failure ultimately leading to premature death.

The pathophysiology of Fontan failure is multifactorial. Both systolic and diastolic ventricular dysfunction are frequently seen, but Fontan failure is also prevalent in patients with preserved ventricular function (21). Elevated PVR plays a critical role in Fontan failure, as increases in PVR cannot be compensated for by a sub-pulmonary ventricle. Even mild elevations in PVR will result in decreased pulmonary blood flow and decreased systemic ventricular preload. Leaving aside the methodological considerations of PVR determination, there is one study that identified Fontan patients with mild elevations in PVR coupled with a low cardiac index as the subgroup at high risk for Fontan failure (2).

Increased PVR aggravates chronic venous congestion, which results in an imbalance of lymphatic homoeostasis. Venous hypertension increases production of lymphatic fluid and simultaneously impairs lymphatic return to the central venous system. This imbalance is considered a contributor to conditions such as protein losing enteropathy or plastic bronchitis, where lymph fluid is leaking into the gut or the lungs, respectively (19).

\section{Pulmonary vasodilator therapy in Fontan patients}

For patients with single ventricle physiology efforts to avoid pulmonary vascular disease start in the neonatal period. Neonatal management of single-ventricle patients has to aim at optimal preparation of the pulmonary artery bed for the Fontan circulation avoiding prolonged periods of pulmonary over-circulation, pulmonary hypertension and ventricular volume load, but, at the same time, it has to allow for sufficient pulmonary artery growth (17). Once the Fontan circulation is established, limitation of cyanosis can be of theoretical benefit as discussed above. Closure of a fenestration or veno-venous collaterals to reduce cyanosis, however, can result in an increase in CVP in turn promoting adverse sequelae of venous congestion.

Once pulmonary vascular disease is considered in patients with a Fontan circulation, pulmonary vasodilator therapies are often initiated. There are several drug classes established for the treatment of idiopathic pulmonary hypertension and pulmonary hypertension associated with congenital heart disease. PDE-5-inhibitors such as sildenafil, tadalafil or udenafil enhance the NO pathway by increasing intracellular 
cyclic GMP levels. Bosentan, macitentan, and ambrisentan are endothelin receptor antagonists that have different receptor affinities: bosentan and macitentan are dualreceptor antagonists, while ambrisentan is relatively selective to the type $\mathrm{A}$ endothelin receptor $\left(\mathrm{ET}_{\mathrm{A}}\right)$. Macitentan shows slow dissociation kinetics and provides non-competitive antagonism at the endothelin receptor.

Hepatotoxicity is a possible side effect of endothelin receptor antagonists and needs to be considered when choosing pulmonary vasodilator therapy in Fontan patients keeping in mind the risk of hepatic dysfunction. The second-generation endothelin receptor antagonists ambrisentan and macitentan are thought to have considerably less hepatic side effects compared to bosentan.

Prostacyclin analogues such as intravenous epoprostenol, inhaled iloprost or subcutaneous treprostinil are very potent in lowering PVR, but their routine use is limited as their application is cumbersome.

Several studies have evaluated the effects of PDE-5inhibitors $(45,64-70)$, endothelin receptor antagonists (71-77) and prostacyclin analogues (78) in Fontan patients. So far, there is no published data on the use of riociguat, a stimulator of the soluble guanylate cyclase, in Fontan patients. Trials investigating combination therapies have not yet been performed.

\section{Prophylactic use of pulmonary vasodilators in Fontan patients}

As PVR modulates pulmonary blood flow and systemic ventricular preload in Fontan patients, pulmonary vasodilators should improve cardiac output and trials that evaluated pulmonary antihypertensive drugs in Fontan patients have therefore focused on markers of improved cardiac output such as exercise capacity, New York Heart Association (NYHA) functional class or quality of life. An improvement of exercise capacity (6-minute walk test, anaerobic threshold, peak $\mathrm{VO}_{2}$, oxygen pulse, cardiopulmonary exercise test time or increase in cardiac index) was found in a number of smaller trials $(45,64,68,73$ $75,77,78)$ while others showed no improvement $(67,69,72)$. Result from various studies have also not been consistent regarding NYHA functional class or quality of life $(45,68,72,73,75-77)$.

Interestingly, two recently published meta-analyses $(79,80)$ yielded conflicting results due to differences in methods. While Li et al. concluded that pulmonary vasodilators appear not to be beneficial in the categories of pulmonary resistance, heart function, or quality of life and that there is no significant evidence to confirm that most pulmonary vasodilators improve exercise capacity in Fontan patients (79), Wang and co-workers found that there was a significant improvement in NYHA functional class, 6-minute walking distance and peak $\mathrm{VO}_{2}(80)$. It is beyond the scope of this article to discuss the inherent methodological problems of systematic meta-analyses, but even if there was a significant effect of pulmonary vasodilators in any of the small randomized controlled trials or the meta-analyses, the effect size was small.

Therefore, it was not surprising, that in the Fontan Udenafil Exercise Longitudinal (FUEL) Trial, a phase III multicentre clinical trial with 400 patients, treatment with the PDE-5-inhibitor udenafil was not associated with the primary outcome, an improvement in oxygen consumption at peak exercise. It was, however, associated with some improvement in markers of exercise performance at the ventilatory anaerobic threshold. But the effect size was again small (70). For Endothelin receptor antagonist a larger clinical study (RUBATO Trial) assessing the efficacy and safety of macitentan in Fontan patients is currently recruiting patients—results have not been published yet.

To date, although safe and well tolerated, there is no evidence that prophylactic pulmonary vasodilator therapy has an impact on prognosis of the entire group of Fontan patients. It is important though to identify subgroups within this patient population that might benefit from such therapies. There is no strong physiological basis for attempting to lower PVR in patients with normal baseline PVR. In a study assessing basal PVR and its response to inhaled NO during cardiac catheterization, there was no significant change in PVR in patients with a baseline PVRi $<2 \mathrm{WU} \times \mathrm{m}^{2}(27)$. Similar results were shown in a study combining cardiac catheterization and MRI. During peak exercise, inhaled NO and $100 \%$ oxygen did not result in a further reduction of normal baseline PVR or a change in cardiac output (6). In a controlled randomized crossover trial, an improvement in oxygen consumption at the anaerobic threshold was only observed in subjects treated with sildenafil who had elevated serum BNP levels (67).

\section{Pulmonary vasodilators in Fontan failure}

Several small cohort studies have reported the effects of treatment with pulmonary vasodilators in symptomatic patients with overt Fontan failure. Treatment with sildenafil improved symptoms of Fontan failure including protein 
losing enteropathy, plastic bronchitis, chylous effusions or severe cyanosis in one study (66), but results have to be interpreted with caution as creation of a fenestration was part of the treatment in 5 of the 13 patients. Morchi and colleagues treated six failing Fontan patients with either persistent cyanosis or effusions with sildenafil (65). In this study, PVR was elevated $\left(>3 \mathrm{WU} \times \mathrm{m}^{2}\right)$ in 5 of 6 patients and sildenafil treatment resulted in increased arterial saturation and lower pulmonary artery pressure and PVR. In another small study with 24 Fontan patients who had a PVRi $\geq 2 \mathrm{WU} \times \mathrm{m}^{2}$, treatment with Bosentan resulted in a reduction of PVR (77). A group from Belgium showed an improvement of oxygen saturations and exercise performance in 5 of their 10 failing Fontan patients treated with Bosentan over a period of 16 weeks (71). These patients deteriorated again when the drug was discontinued. Thus, while there was no significant treatment effect for the entire group, there was a subgroup that had a benefit from bosentan treatment.

\section{Conclusions}

PVR is one of the main determinants of cardiac output in the Fontan patient. Pathophysiological considerations support the use of pulmonary vasodilators in patients with Fontan failure and elevated PVR, but scientific evidence is scarce. Current published experience does not support proactive pulmonary vasodilator therapy in well-functioning Fontan patients with low pulmonary arterial pressure and low PVR.

Still, there is some evidence suggesting that chronic pulmonary vascular processes lead to progressive elevation of PVR in most Fontan patients. It will be important to understand the molecular mechanisms involved in these chronic vascular processes to improve the longterm outcome of Fontan patients. Perhaps identification of the molecular targets will help to modulate Fontan physiology and avoid or ameliorate maladaptive processes in the Fontan circulation. Future research should focus on identification of better parameters to characterize the functional status of Fontan patients, on selection criteria for pharmacological and non-pharmacological therapies aiming at an improvement of functional status, and on the incorporation of new drugs into our armamentarium.

\section{Acknowledgments}

Funding: None.

\section{Footnote}

Provenance and Peer Review: This article was commissioned by the Guest Editors (Christian Apitz and Astrid Lammers) for the series "Pediatric Pulmonary Hypertension" published in Cardiovascular Diagnosis and Therapy. The article has undergone external peer review.

Conflicts of Interest: The authors have completed the ICMJE uniform disclosure form (available at http://dx.doi. org/10.21037/cdt-20-431). The series "Pediatric Pulmonary Hypertension" was commissioned by the editorial office without any funding or sponsorship. The authors have no other conflicts of interest to declare.

Ethical Statement: The authors are accountable for all aspects of the work in ensuring that questions related to the accuracy or integrity of any part of the work are appropriately investigated and resolved.

Open Access Statement: This is an Open Access article distributed in accordance with the Creative Commons Attribution-NonCommercial-NoDerivs 4.0 International License (CC BY-NC-ND 4.0), which permits the noncommercial replication and distribution of the article with the strict proviso that no changes or edits are made and the original work is properly cited (including links to both the formal publication through the relevant DOI and the license). See: https://creativecommons.org/licenses/by-nc-nd/4.0/.

\section{References}

1. Khairy P, Fernades SM, Landzberg MJ, et al. Long term survival, modes of death, and predictors of mortality in patients with Fontan surgery. Circulation 2008;117:85-92.

2. Egbe AC, Conolly HM, Borlaug BA, et al. Hemodynamics of Fontan failure - The role of pulmonary vascular disease. Circ Heart Fail 2017;10:e004515.

3. Egbe AC, Reddy YNV, Borlaug BA, et al. Venous congestion and pulmonary vascular function in Fontan circulation: Implication for prognosis and treatment. Int J Cardiol 2018;271:312-6.

4. Mori M, Aguirre AJ, Book WM, et al. Beyond a broken heart: Circulatory dysfunction in the failing Fontan. Pediatr Cardiol 2014;35:569-79.

5. d'Udekem Y, Iyengar AJ, Winlaw DS, et al. Redefining expectations of long-term survival after the Fontan procedure: twenty-five years of follow-up from the entire population of Australia and New Zealand. Circulation 
2014;130:S32-S38.

6. Pushparajah K, Wong JK, Razavi R, et al. Magnetic resonance imaging catheter stress haemodynamics postFontan in hypoplastic left heart syndrome. Eur Heart J Cardiovasc Imaging 2016;17:644-51.

7. Möller P, Weitz M, Uebing A, et al. Exercise capacity of a contemporary cohort of children with hypoplastic left heart syndrome after staged palliation. Eur J Cardiothorac Surg 2009;36:980-5.

8. Diller GP, Giardini A, Hager A, et al. Predictors of morbidity and mortality in contemporary Fontan patients: results from a multicenter study including cardiopulmonary exercise testing in 321 patients. Eur Heart J 2010;31:3073-83.

9. Udholm S, Aldweib N, Veldtman GR, et al. Prognostic power of cardiopulmonary exercise testing in Fontan patients: a systematic review. Open Heart 2018;5:e000812.

10. Goldstein BH, Connor CE, Rocchini AP, et al. Relation of systemic venous return, pulmonary vascular resistance, and diastolic dysfunction to exercise capacity in patients with single ventricle receiving Fontan palliation. Am J Cardiol 2010;105:1169-75.

11. Van De Bruaene A, La Gerche A, Budts W, et al. Sildenafil improves exercise hemodynamics in Fontan patients. Circ Cardiovasc Imaging 2014;7:265-73.

12. Presson RG, Baumgartner WA, Wagner WW, et al. Pulmonary capillaries are recruited during pulsatile flow. J Appl Physiol 2002;92:1183-90.

13. Lemler MS, Scott WA, Ramaciotti C, et al. Fenestration improves clinical outcome of the Fontan procedure: a prospective, randomized study. Circulation 2002;105:207-12.

14. Fan F, Liu Z, Wang Q, et al. Effect of Fenestration on early postoperative outcome in extracardiac Fontan patients with different risk levels. Pediatr Cardiol 2017;38:643-9.

15. Atz AM, Travison TG, Gersony WM, et al. Late status of Fontan patients with persistent surgical fenestration. J Am Coll Cardiol 2011;57:2437-43.

16. Bouhout I, Ben-Ali W, Poirier N, et al. Effect of fenestration on Fontan procedure outcomes: a metaanalysis and review. Ann Thorac Surg 2020;109:1467-74.

17. Gewillig M, Brown SC, Gorenflo M, et al. Volume load paradox while preparing for the Fontan: not too much for the ventricle, not too little for the lungs. Interact Cardiovasc Thorac Surg 2010;10:262-5.

18. Schlangen J, Fischer G, Uebing A, et al. Arterial elastance and its impact on intrinsic right ventricular function in palliated hypoplastic left heart syndrome. Int J Cardiol 2013;168:5385-9.

19. Rychik J. The relentless effects of the Fontan paradox. Semin Thorac Cardiovasc Surg Pediatr Card Surg Annu 2016;19:37-43.

20. Goldberg DJ, Dodds K, Rychik J. Rare problems associated with the Fontan circulation. Cardiol Young 2010;20:113-9.

21. Book WM, Gerardin J, Rodriguez F, et al. Clinical Phenotypes of Fontan Failure: Implications for Management. Congenit Heart Dis 2016;11:296-308.

22. Ridderbos FJ, Wolff D, Berger RM, et al. Adverse pulmonary vascular remodeling in the Fontan circulation. J Heart Lung Transplant 2015;34:404-13.

23. Schmitt B, Steendijk P, Kuehne T, et al. Pulmonary vascular resistance, collateral flow, and ventricular function in patients with a Fontan circulation at rest and during dobutamine stress. Circ Cardiovasc Imaging 2010;3:623-31.

24. Pushparajah K, Tzifa A, Razavi R et al. Cardiovascular magnetic resonance catheterization derived pulmonary vascular resistance and medium-term outcomes in congenital heart disease. J Cardiovasc Magn Reson 2015;17:28.

25. Simonneau G, Montani D, Celermajer DS, et al. Haemodynamic definitions and updated clinical classification of pulmonary hypertension. Eur Respir J 2019;53:1801913

26. Hansmann G, Koestenberger M, Zartner P, et al. 2019 updated consensus statement on the diagnosis and treatment of pediatric pulmonary hypertension: the European Pediatric Pulmonary Disease Network (EPPVDN), endorsed by AEPC, ESPR and ISHLT. J Heart Lung Transplant 2019;38:879-901.

27. Khambadkone S, Li J, Redington AN, et al. Basal pulmonary vascular resistance and nitric oxyde responsiveness late after Fontan-type operation. Circulation 2003;107:3204-8.

28. Mitchell MB, Campbell DN, Coll JR, et al. Evidence of pulmonary vascular disease after heart transplantation for Fontan circulation failure. J Thorac Cardiovasc Surg 2004;128:693-702.

29. Ishida H, Kogaki S, Ozono K, et al. Attenuation of bone morphogenetic protein receptor type 2 expression in the pulmonary arteries of patients with failed Fontan circulation. J Thorac Cardiovasc Surg 2012;143:e24-6.

30. Zongtao Y, Huishan W, Hongguang H, et al. Experimental study of nonpulsatile flow perfusion and structural 
remodeling of pulmonary microcirculatory vessels. Thorac Cardiovasc Surg 2010;58:468-72.

31. Henaine R, Vergnat M, Serraf A, et al. Effects of lack of pulsatility on pulmonary endothelial function in the Fontan circulation. J Thorac Cardiovasc Surg 2013;146:522-9.

32. Henaine R, Vergnat M, Bacha E, et al. Hemodynamics and arteriovenous malformations in cavopulmonary anastomosis: The case for residual antegrade pulsatile flow. J Thorac cardiovasc Surg 2013;146:1359-65.

33. Kurotobi S, Sano T, Okada S, et al. Bidirectional cavopulmonary shunt with right ventricular outflow patency: The impact of pulsatility on pulmonary endothelial function. J Thorac Cardiovasc Surg 2001;121:1161-8.

34. Siragusa M, Fleming I. The eNOS signalosome and its link to endothelial dysfunction. Pflugers Arch 2016;468:1125-37.

35. Latus H, Lederle A, Apitz A, et al. Evaluation of pulmonary endothelial function in Fontan patients. J Thorac Cardiovasc Surg 2019;158:523-531.e1.

36. Sage E, Mercier O, Fadel E, et al. Right lung ischemia induces contralateral pulmonary vasculopathy in an animal model. J Thorac Cardiovasc Surg 2012;143:967-73.

37. Brown JW, Ruzmetov M, Turrentine MW, et al. Pulmonary arteriovenous malformations in children after the Kawashima operation. Ann Thorac Surg 2005;80:1592-6.

38. Field-Ridley A, Heljasvaara R, Fineman JR, et al. Endostatin, an inhibitor of angiogenesis, decreases after bidirectional superior cavo-pulmonary anastomosis. Pediatr Cardiol 2013;34:291-5.

39. Vettukattil JJ. Is the hepatic factor a miRNA that maintains the integrity of pulmonary microvasculature by inhibiting the vascular endothelial growth factor? Curr Cardiol Rev 2017;13:244-50.

40. Dengler VL, Galbraith M, Espinosa JM. Transcriptional regulation by hypoxia inducible factors. Crit Rev Biochem Mol Biol 2014;49:1-15.

41. Prabhakar NR, Semenza GL. Adaptive and maladaptive cardiorespiratory responses to continuous and intermittend hypoxia mediated by hypoxia-inducible factors 1 and 2 . Physiol Rev 2012;92:967-1003.

42. Laddha AP, Kulkarni YA. VEGF and FGF-2: promising targets for the treatment of respiratory disorders. Respir Med 2019;156:33-46.

43. Semenza GL. Hypoxia-inducible factors: mediators of cancer progression and targets for cancer therapy. Trends Pharmacol Sci 2012;33:207-14.
44. Tomkiewicz-Pajak L, Plazak W, Podolec P, et al. Iron deficiency and hematological changes in adult patients after Fontan operation. J Cardiol 2014;64:384-9.

45. Mori H, Park IS, Nakanishi T, et al. Sildenafil reduces pulmonary vascular resistance in single ventricular physiology. Int J Cardiol 2016;221:122-7.

46. Suda K, Matsumura M, Matsumoto M, et al. Increased vascular endothelial growth factor in patients with cyanotic congenital heart diseases may not be normalized after a Fontan type operation. Ann Thorac Surg 2004;78:942-6; discussion 946-7.

47. Lévy M, Danel C, Israel-Biet D, et al. Nitric oxide synthase expression by pulmonary arteries: A predictive marker of Fontan procedure outcome? J Thorac Cardiovasc Surg 2003;125:1083-90.

48. Ishida H, Kogaki S, Ozono K, et al. Overexpression of endothelin-1 and endothelin receptors in the pulmonary arteries of failed Fontan patients. Int J Cardiol 2012;159:34-9.

49. Hiramatsu T, Imai Y, Nakazawa M, et al. Time course of Endothelin-1 and Adrenomedullin after the Fontan procedure. Ann Thorac Surg 1999;68:169-72.

50. Yamagishi M, Kurosawa H, Kitamura N, et al. The role of plasma endothelin in the Fontan circulation. J Cardiovasc Surg (Torino) 2002;43:793-7.

51. Bocca C, Novo E, Miglietta A, et al. Angiogenesis and fibrogenesis in chronic liver disease. Cell Mol Gastroenterol Hepatol 2015;1:477-88.

52. Deshpande SR, Patel P, Clifton MS, et al. Retroperitoneal paraganglioma in a patient with Fontan: The hypoxia connection. Ann Pediatr Cardiol 2018;11:197-200.

53. Song MK, Kim GB, Won JK, et al. Pheochromocytoma and paraganglioma in Fontan patients: Common more than expected. Congenit Heart Dis 2018;13:608-16.

54. Lin D, Wu J. Hypoxia inducible factor in hepatocellular carcinoma: A therapeutic target. World J Gastroenterol 2015;21:12171-8.

55. Komatsu H, Inui A, Fujisawa T, et al. Liver disease secondary to congenital heart disease in children. Expert Rev Gastroenterol Hepatol 2019;13:651-66.

56. Chen C, Lou T. Hypoxia inducible factors in hepatocellular carcinoma. Oncotarget 2017;8:46691-703.

57. Hirota K. An intimate crosstalk between iron homeostasis and oxygen metabolism regulated by the hypoxia-inducible factors (HIFs). Free Radic Biol Med 2019;133:118-29.

58. Smith TG, Balanos GM, Robbins PA, et al. The increase in pulmonary arterial pressure caused by hypoxia depends on iron status. J Physiol 2008;586:5999-6005. 
59. Smith TG, Talbot NP, Robbins PA, et al. Effects of iron supplementation and depletion on hypoxic pulmonary hypertension. JAMA 2009;302:1444-50.

60. Rhodes CJ, Wharton J, Wilkins MR, et al. Iron deficiency in pulmonary arterial hypertension: a potential therapeutic target. Eur Respir J 2011;38:1453-60.

61. Harvey LD, Chan SY. Emerging metabolic therapies in pulmonary arterial hypertension. J Clin Med 2017;6:e43.

62. Dai Z, Zhu MM, Zhao YY, et al. Therapeutic targeting of vascular remodeling and right heart failure in pulmonary arterial hypertension with a HIF-2 $\alpha$ inhibitor. Am J Respir Crit Care Med 2018;198:1423-34.

63. Luo Y, Teng X, Yan X, et al. CD146-HIF1 hypoxic reprogramming drives vascular remodelling and pulmonary arterial hypertension. Nat Commun 2019;10:3551.

64. Giardini A, Balducci A, Picchio FM, et al. Effect of sildenafil on haemodynamic response to exercise and exercise capacity in Fontan patients. Eur Heart J 2008;29:1681-7.

65. Morchi GS, Ivy DD, Kay J, et al. Sildenafil increases systemic saturation and reduces pulmonary artery pressure in patients with failing Fontan physiology. Congenit Heart Dis 2009; 4:107-11.

66. Reinhardt Z, Uzun O, Stumper O, et al. Sildenafil in the management of the failing Fontan circulation. Cardiol Young 2010;20:522-5.

67. Goldberg DJ, French B, Rychik J, et al. Impact of oral sildenafil on exercise performance in children and young adults after the Fontan operation: a randomized, doubleblind, placebo-controlled, crossover trial. Circulation 2011;123:1185-93.

68. Sabri MR, Zolfi-Gol A, Haghjooy-Javanmard S, et al. Effect of tadalafil on myocardial and endothelial function and exercise performance after modified Fontan operation. Pediatr Cardiol 2016;37:55-61.

69. Goldberg DJ, Zak V, Paridon SM, et al. Results of a phase I/II multi-center investigation of udenafil in adolescents after Fontan palliation. Am Heart J 2017;188:42-52.

70. Goldberg DJ, Zak V, Paridon SM, et al. Results of the FUEL Trial. Circulation 2020;141:641-51.

Cite this article as: Becker $\mathrm{K}$, Uebing A, Hansen JH. Pulmonary vascular disease in Fontan circulation-is there a rationale for pulmonary vasodilator therapies? Cardiovasc Diagn Ther 2021;11(4):1111-1121. doi: 10.21037/cdt-20-431
71. Ovaert C, Thijs D, Mertens L, et al. The effect of bosentan in patients with a failing Fontan circulation. Cardiol Young 2009;19:331-9.

72. Schuuring MJ, Vis JC, Bouma BJ, et al. Impact of bosentan on exercise capacity in adults after the Fontan procedure: a randomized controlled trial. Eur J Heart Fail 2013;15:690-8.

73. Hebert A, Mikkelsen UR, Sondergaard L, et al. Bosentan improves exercise capacity in adolescents and adults after Fontan operation: the TEMPO (Treatment with Endothelin receptor antagonist in Fontan patients, a randomized, placebo-controlled, double-blind study Measuring Peak Oxygen consumption) study. Circulation 2014;130:2021-30.

74. Derk G, Houser L, Aboulhosn J, et al. Efficacy of endothelin blockade in adults with Fontan physiology. Congenit Heart Dis 2015:10:E11-E16.

75. Cedars AM, Saef J, Ludbrook PA, et al. Effect of ambrisentan on exercise capacity in adult patients after the Fontan procedure. Am J Cardiol 2016;117:1524-32.

76. Shang XK, Lu R, Dong NG, et al. Efficacy of bosentan in patients after Fontan procedures: a double-blind, randomized controlled trial. J Huazhong Univ Sci Technolog Med Sci 2016;36:534-40.

77. Agnoletti G, Gala S, Bergamasco L, et al. Endothelin inhibitors lower pulmonary vascular resistance and improve functional capacity in patients with Fontan circulation. J Thorac Cardiovasc Surg 2017;153:1468-75.

78. Rhodes J, Ubeda-Tikkanen A, Landzberg MJ, et al. Effect of inhaled iloprost on the exercise function of Fontan patients: A demonstration of concept. Int J Cardiol 2013;168:2435-40.

79. Li D, Zhou X, An Q, et al. Pulmonary vasodilator therapy after the Fontan procedure: a meta-analysis. Heart Fail Rev 2021;26:91-100.

80. Wang $W, H u X$, Huang $W$, et al. The efficacy and safety of pulmonary vasodilators in patients with Fontan circulation: a meta-analysis of randomized controlled trials. Pulm Circ 2019;9:2045894018790450. 